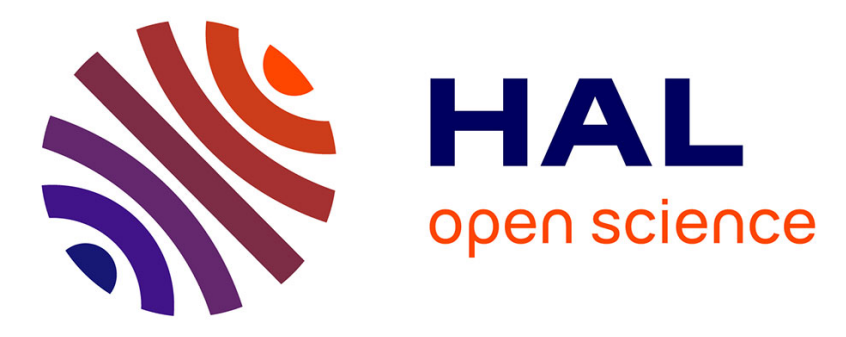

\title{
Illumination-robust multispectral demosaicing
}

Sofiane Mihoubi, Benjamin Mathon, Jean-Baptiste Thomas, Olivier Losson, Ludovic Macaire

\section{To cite this version:}

Sofiane Mihoubi, Benjamin Mathon, Jean-Baptiste Thomas, Olivier Losson, Ludovic Macaire. Illumination-robust multispectral demosaicing. 7th IEEE International Conference on Image Processing Theory, Tools and Applications (IPTA 2017), Nov 2017, Montreal, Canada. pp.1-6, 10.1109/IPTA.2017.8310135 . hal-01681427

\section{HAL Id: hal-01681427 https://hal.science/hal-01681427}

Submitted on 19 Jan 2018

HAL is a multi-disciplinary open access archive for the deposit and dissemination of scientific research documents, whether they are published or not. The documents may come from teaching and research institutions in France or abroad, or from public or private research centers.
L'archive ouverte pluridisciplinaire HAL, est destinée au dépôt et à la diffusion de documents scientifiques de niveau recherche, publiés ou non, émanant des établissements d'enseignement et de recherche français ou étrangers, des laboratoires publics ou privés. 


\section{Illumination-robust multispectral demosaicing}

Sofiane Mihoubi ${ }^{1}$, Benjamin Mathon ${ }^{1}$, Jean-Baptiste Thomas ${ }^{2,3}$, Olivier Losson ${ }^{1}$ and Ludovic Macaire ${ }^{1}$

${ }^{1}$ Univ. Lille, CNRS, Centrale Lille, UMR 9189 - CRIStAL -

Centre de Recherche en Informatique Signal et Automatique de Lille, F-59000 Lille, France

e-mail: sofiane.mihoubi@ed.univ-lille1.fr, \{benjamin.mathon, olivier.losson, ludovic.macaire\}@univ-lille1.fr

2 Le2i, FRE CNRS 2005, Université Bourgogne Franche-Comté, 21000 Dijon, France

3 Norwegian University of Science and Technology, Norwegian Colour and Visual Computing Laboratory, Gjøvik, Norway e-mail: jean.b.thomas@ntnu.no 


\begin{abstract}
Snapshot multispectral cameras that are equipped with filter arrays acquire a raw image that represents the radiance of a scene over the electromagnetic spectrum at video rate. These cameras require a demosaicing procedure to estimate a multispectral image with full spatio-spectral definition. Such a procedure is based on spectral correlation properties that are sensitive to illumination. In this paper, we first highlight the influence of illumination on demosaicing performances. Then we propose camera-, illumination-, and raw image-based normalisations that make demosaicing robust to illumination. Experimental results on state-of-the-art demosaicing algorithms show that such normalisations improve the quality of multispectral images estimated from raw images acquired under various illuminations.
\end{abstract}

\title{
Index Terms
}

Image demosaicing, Multispectral filter array, Illumination, Spectral correlation, Snapshot camera.

\section{INTRODUCTION}

Digital multispectral cameras sample the electromagnetic spectrum into several spectral bands. Such devices provide multispectral images that represent the scene radiance of each spectral band as a separate channel. Multispectral images are acquired in different illumination conditions for various application fields such as medical imaging [1], precision agriculture [2], art studies [3], vision inspection for food quality control [4] or waste sorting [5]. The information available in each channel of a multispectral image results from a spectral integration of the product between the scene reflectance, illumination, camera filter transmittances and sensor sensitivity. Therefore, multispectral images are dependent upon the illumination properties and the camera spectral sensitivity.

For nearly twenty years, multispectral images have been acquired using sequential technologies, i.e., one spectral band or one single pixel row at a time [6]. Recently snapshot multispectral cameras have emerged to acquire all spectral bands in a single shot [7]. In particular, a single-sensor snapshot technology uses a multispectral filter array (MSFA) [8,9] and provides a raw image in which each pixel is characterised by the level of a single spectral component. The component levels that miss at each pixel can be estimated by a process known as demosaicing to recover all channels in full definition. Demosaicing relies on two properties of multispectral images, namely spatial correlation between spatially close pixels within a channel, and spectral correlation between levels of different components at the same pixel [10].

In this paper, we study the impact of the illumination on spectral correlation and its influence on demosaicing performances. We observe that spectral correlation decreases when the illumination becomes non-homogeneous over the spectrum. Then we propose a method that adjusts channel levels before demosaicing, which improves demosaicing robustness to illumination variations. This method uses normalisation factors that either depend on the camera spectral sensitivity only (camera-based normalisation), on both the sensitivity and the illumination (camera- and illumination-based normalisation), or on the statistics extracted from the acquired raw image (raw image-based normalisation).

This paper is organised as follows. In Sec. II we simulate the acquisition of fully-defined multispectral 
images under various illuminations. These images are then used to simulate raw images according to a given MSFA. In Sec. III we shortly review multispectral demosaicing methods and present their limitations when illumination changes. In Sec. IV we propose camera-based, camera- and illumination-based, and raw imagebased normalisations that are robust to illumination. Finally, we experimentally show the impacts of these normalisations on demosaicing performances in Sec. V.

\section{RAW IMAGE SIMULATION}

To assess demosaicing performances, fully-defined multispectral images are required to serve as references although they cannot be provided by single-sensor multispectral cameras. In this section, we simulate the acquisition of reference multispectral images, then we sample them to form raw images that will undergo demosaicing.

\section{A. Multispectral image simulation}

Let us consider the multispectral image $\mathbf{I}=\left\{I^{k}\right\}_{k=1}^{K}$ made of $K$ fully-defined channels associated to $K$ spectral bands. Assuming ideal optics and spatially homogeneous illumination over the sensor, the level $I_{p}^{k}$ of channel $I^{k}$ at a pixel $p$ is given by a simple image formation model as:

$$
I_{p}^{k}=Q\left(\int_{\Omega} E(\lambda) \cdot R_{p}(\lambda) \cdot S^{k}(\lambda) d \lambda\right),
$$

where $Q$ is a quantization function of the received energy, $\Omega$ is the considered spectral range, $E(\lambda)$ is the illumination, $R_{p}(\lambda)$ is the reflectance of the surface element observed at pixel $p$, and $S^{k}(\lambda)$ is the spectral response curve (SRC) of the camera for the band $k$ (combining the filter transmittance and sensor sensitivity).

To simulate reference multispectral images from this model, we consider:

- Four CIE standard illuminants (E, D65, F12 and A) and two real illuminations (Paulmann $2900 \mathrm{~K}$ halogen lamps (HA) and Advanced Illumination DL097-WHIIC LED diffuse dome (LD)) whose relative power spectral distributions $E(\lambda)$ are defined for all $\lambda \in \Omega \doteq[400 \mathrm{~nm}, 700 \mathrm{~nm}]$ (see Fig. 1(a)).

- The reflectance data provided by CAVE [11] and FOSTER [12] databases that respectively represent 32 scenes of real-world materials and objects acquired in indoor conditions and 8 natural scenes acquired in outdoor conditions. Each scene is acquired over 31 spectral bands of width $10 \mathrm{~nm}$ and evenly centred at $\lambda \in\{400 \mathrm{~nm}, \ldots, 700 \mathrm{~nm}\}$, leading to 31-channel images. By associating each channel with its band centre and assuming linear continuity of reflectance, we get the reflectance $R_{p}(\lambda)$ for each pixel $p$ and all integer $\lambda \in \Omega$ using linear interpolation.

- The spectral sensitivity of IMEC's "snapshot mosaic" multispectral camera [13] (shortly called IMEC camera in the following) that is among the very few ones of the same kind available on the market up to now. IMEC camera segments $\Omega$ into $K=16$ spectral bands. Each band $k \in \mathcal{B} \doteq\{1, \ldots, K\}$ is acquired according to the associated SRC $S^{k}(\lambda)$ centred at $\lambda^{k} \in \Lambda \doteq\{469,480,489,499,513,524,537,551,552,566,580,590$, $602,613,621,633\}$ (in nanometres). To avoid most spectral artefacts, the optical device is equipped with a $450-650 \mathrm{~nm}$ band-pass filter (see Fig. 1(b)). 


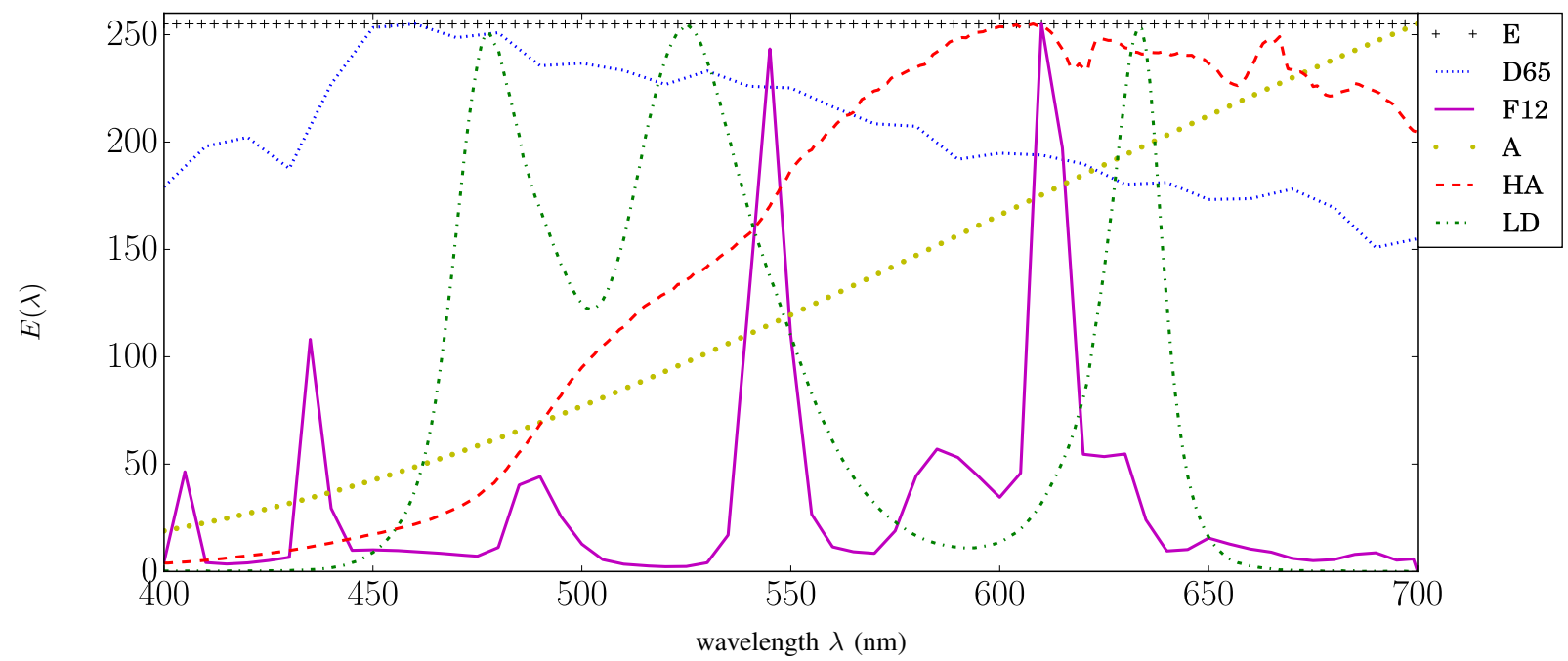

(a)

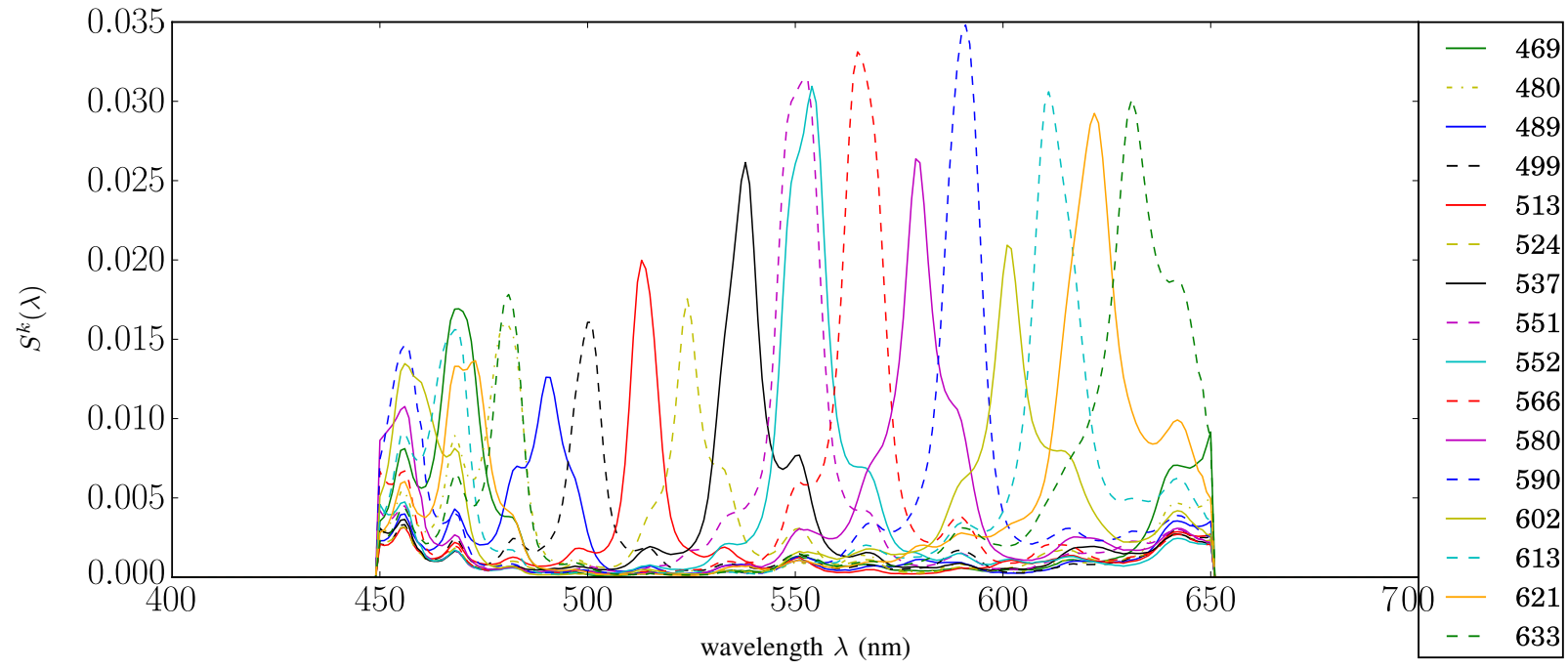

(b)

Fig. 1. Image simulation data: (a) Relative spectral power distributions of CIE E, D65, A, and F12 illuminants and of HA and LD real illuminations (acquired with Avantes AvaSpec-3648 spectrometer). (b) IMEC camera SRCs (caption: centre wavelengths $\lambda^{k} \in \Lambda$ in ascending order).

The 16 reference channels simulated according to IMEC camera SRCs are obtained from Eq. (1) by a discrete sum with $d \lambda=1$. $E(\lambda), R_{p}(\lambda)$, and $S^{k}(\lambda)$ are real-valued functions and $Q$ quantizes the energy on 8 bits so that $0 \leq I_{p}^{k} \leq 2^{8}-1$. To prevent saturation in the simulated images, the illumination $E(\lambda)$ is scaled to reach 255 at its maximum over $\Omega$, and SRCs are scaled so that $\max _{l \in \mathcal{B}} \sum_{\lambda \in \Omega} S^{l}(\lambda)=1$. Reflectance values range between 0 and 1, FOSTER reflectance values superior to 1 being truncated. For each of the six illuminations, this simulation overall provides 32 (CAVE) and 8 (FOSTER) 16-channel multispectral images of size $512 \times 512$ $(\mathrm{CAVE})$ and $1344 \times 1024$ pixels $($ FOSTER). 


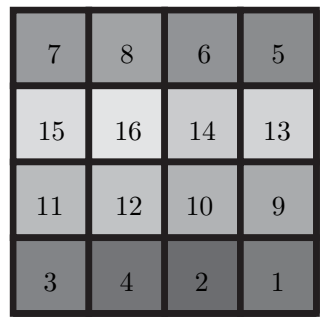

Fig. 2. Basic MSFA pattern of IMEC camera. Note that bands (shown indices) are not arranged in ascending order of the classical pixel read-out due to manufacturing constraints.

\section{B. Raw image simulation and demosaicing}

MSFAs are defined by a basic repetitive pattern that samples each spectral band at least once. For instance, the $4 \times 4$ basic pattern of IMEC camera samples each band exactly once as shown in Fig. 2. Single-sensor multispectral cameras provide a raw image $I^{\text {raw }}$ in which each pixel $p$ is associated with a single component $k \in \mathcal{B}$ according to the MSFA pattern. Let $\mathcal{A}$ be the set of all pixels and $\mathcal{A}^{k}$ be the pixel subset where the MSFA samples the band $k$, such that $\mathcal{A}=\bigcup_{k \in \mathcal{B}} \mathcal{A}^{k}$. Then, $I^{\text {raw }}$ can be simulated from the reference fully-defined image $\mathbf{I}=\left\{I^{k}\right\}_{k \in \mathcal{B}}$ by spectral sampling according to the location of $p$ : for all $p \in \mathcal{A}^{k}, I_{p}^{r a w}=I_{p}^{k}$.

To obtain an estimated multispectral image $\hat{\mathbf{I}}$ with $K$ fully-defined channels, the demosaicing procedure estimates the $K-1$ levels that miss at each pixel in $I^{r a w}$ : for all $p \in \mathcal{A}^{k}, \hat{\mathbf{I}}_{p}=\left(\hat{I}_{p}^{1}, \ldots, \hat{I}_{p}^{k-1}, I_{p}^{k}, \hat{I}_{p}^{k+1}, \ldots, \hat{I}_{p}^{K}\right)$, where $\hat{I}_{p}^{i}, i \neq k$, is the estimated level of channel $I^{i}$ at $p$.

\section{DEMOSAICING AND SPECTRAL CORRELATION LIMITATIONS}

Gunturk et al. [14] show that the three channels of natural colour images are strongly correlated. By an analysis of the wavelet coefficients, Lian et al. [15] confirm that the spatial high-frequency information carried by the different channels are not only strongly correlated but almost identical. This spectral correlation is often used to find the missing components at a given pixel in colour demosaicing schemes [16]. Multispectral demosaicing uses it in different methods that we present in Sec. III-A. The extension of spectral correlation to the multispectral domain however ignores its sensitivity to illumination. That leads us to highlight the effect of illumination changes on spectral correlation in Sec. III-B.

\section{A. Multispectral demosaicing methods}

Only few methods in the literature are suitable to demosaic raw images provided by IMEC camera. The most intuitive one computes a weighted average of the available levels in the neighbourhood of each pixel. Such weighted bilinear (WB) interpolation uses spatial correlation only. Brauers and Aach [17] take spectral correlation into account in a demosaicing algorithm based on the spectral channel difference (SD) planes. Mihoubi et al. [10] use the pseudo-panchromatic image (PPI) defined as the average of all channels of a multispectral image. Showing that spectral correlation between a given channel and the PPI is stronger than between-channel correlation, they estimate the PPI from the raw image and propose a demosaicing scheme based 
on PPI differences (PPID). They also extend the binary tree-based edge-sensing (BTES) [18] and discrete wavelet transform (DWT) [19] demosaicing methods to IMEC MSFA in PPIBTES [20] and PPIDWT [10]. These methods respectively use the edge and high-frequency information of the estimated PPI to interpolate the missing levels of each channel. Shinoda et al. [21] propose a multispectral local directional interpolation (MLDI) that combines BTES edge-sensing mechanism with SD spectral correlation implementation.

\section{B. Spectral correlation and illumination}

To highlight the effect of spectrally non-uniform illumination on spectral correlation, we compute the correlation coefficient between the high-frequency information of each channel pair [14, 15]. For this purpose, we apply a circular high-pass filter with a cut-off spatial frequency of 0.25 cycle/pixel on the 2D Fourier transform of each channel. The Pearson correlation coefficient between two high-pass filtered channels $\tilde{I}^{i}$ and $\tilde{I}^{j},(i, j) \in \mathcal{B}^{2}$, is given by:

$$
C\left(\tilde{I}^{i}, \tilde{I}^{j}\right)=\frac{\sum_{p \in \mathcal{A}}\left(\tilde{I}_{p}^{i}-\mu^{i}\right)\left(\tilde{I}_{p}^{j}-\mu^{j}\right)}{\sqrt{\sum_{p \in \mathcal{A}}\left(\tilde{I}_{p}^{i}-\mu^{i}\right)^{2}} \sqrt{\sum_{p \in \mathcal{A}}\left(\tilde{I}_{p}^{j}-\mu^{j}\right)^{2}}},
$$

where $\mu^{i}$ is the mean level of $\tilde{I}^{i}$. For each illumination we compute the average correlation coefficient $\mu_{C}$ over all possible channel pairs and the standard deviation $\sigma_{C}$ of the correlation coefficient.

Table 1. Correlation average and standard deviation (averages over all 32 CAVE (a) and 8 FOSTER (b) simulated images).

(a) CAVE

\begin{tabular}{|c|c|c|c|c|c|c|}
\hline & $\mathrm{E}$ & $\mathrm{D} 65$ & $\mathrm{~F} 12$ & $\mathrm{~A}$ & HA & LD \\
\hline$\mu_{C}$ & 0.894 & 0.884 & 0.514 & 0.785 & 0.814 & 0.724 \\
\hline$\sigma_{C}$ & 0.040 & 0.043 & 0.166 & 0.086 & 0.081 & 0.092 \\
\hline
\end{tabular}

(b) FOSTER

\begin{tabular}{|c|c|c|c|c|c|c|}
\hline & $\mathrm{E}$ & $\mathrm{D} 65$ & $\mathrm{~F} 12$ & $\mathrm{~A}$ & $\mathrm{HA}$ & $\mathrm{LD}$ \\
\hline$\mu_{C}$ & 0.681 & 0.665 & 0.342 & 0.575 & 0.609 & 0.495 \\
\hline$\sigma_{C}$ & 0.104 & 0.106 & 0.192 & 0.133 & 0.132 & 0.139 \\
\hline
\end{tabular}

Table 1 shows the correlation and its dispersion on average over all 32 (CAVE) or 8 (FOSTER) simulated images for each illumination of Fig. 1(a). These results show that the illuminations whose spectral power distribution is uniform over $\Omega$ (E) or can be considered as such (D65) provide channels with the highest and less scattered spectral correlations. The illuminations A and HA, for which $E(\lambda)$ increases with respect to $\lambda$ over $\Omega$, provide channels with lower and more scattered spectral correlations. The illuminations F12 and $\mathrm{LD}$, for which $E(\lambda) \approx 0$ except for three marked peaks, provide channels with the lowest and most scattered spectral correlations.

The spectral power distribution of the illumination therefore strongly affects image spectral correlation, which may affect the performance of demosaicing procedures that rely on this property. In the next section we propose several ways to overcome this issue.

\section{ILLUMinATION-ROBUSt DEMOSAICING}

\section{A. Normalisation steps for demosaicing}

After a first study of the influence of illumination non-uniformity on the channel level ranges, Mihoubi et al. [10] use pre- and post-normalisation steps for demosaicing, which improves the estimation of the PPI 


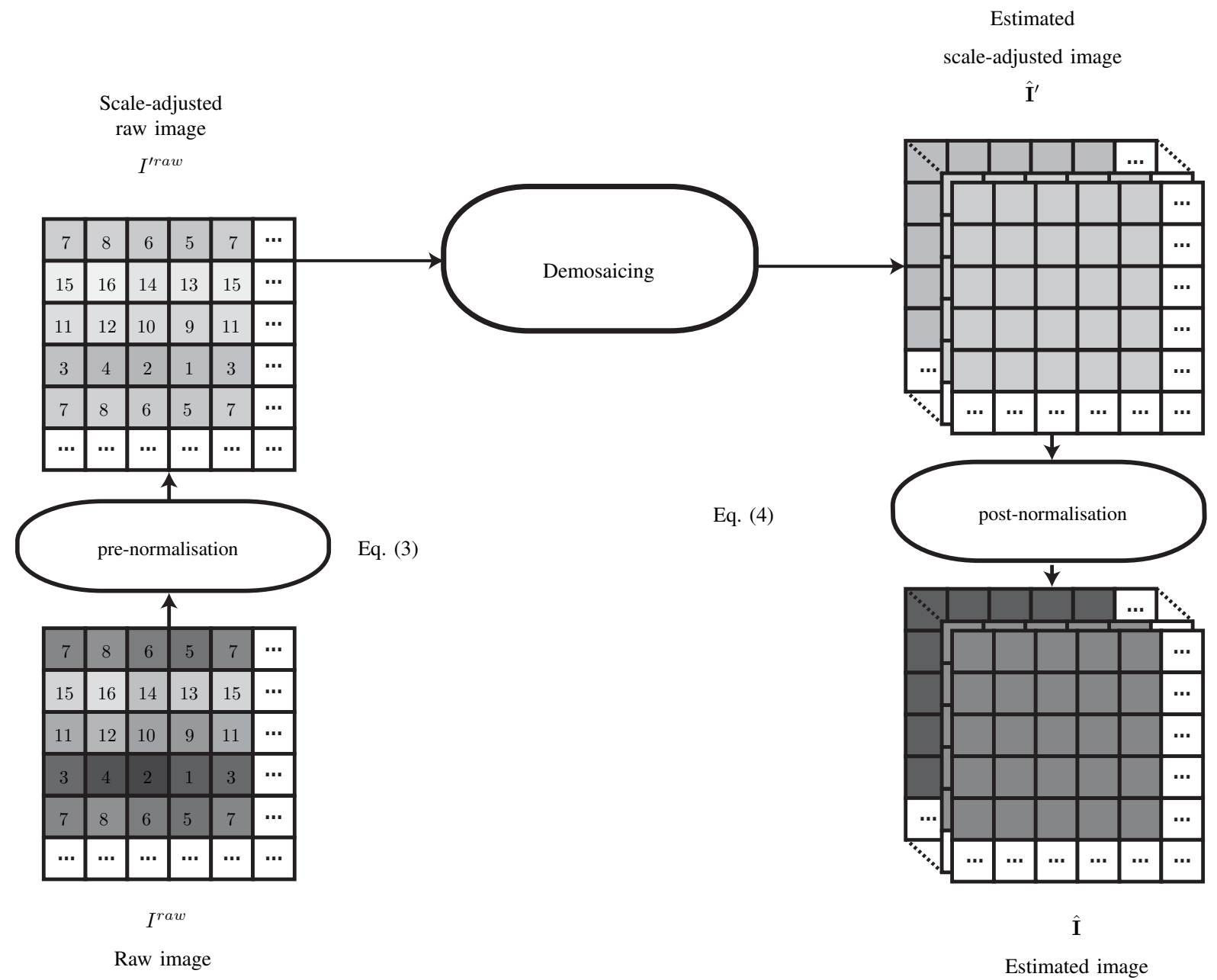

Fig. 3. Normalisation steps for demosaicing.

under various illuminations. Here, we investigate these procedures in depth by proposing new normalisation approaches and by extending them to the demosaicing methods presented in Sec. III-A.

Figure 3 illustrates these normalisation steps. Before demosaicing, we adjust the level scale of each channel by computing a new raw level $I_{p}^{\prime r a w}$ at each pixel $p$. For this purpose, the pre-normalisation step normalises the raw image at each pixel subset $\left\{\mathcal{A}^{k}\right\}_{k \in \mathcal{B}}$ by a specific factor $\rho_{*}^{k}$ as:

$$
I_{p}^{\text {raw }}=\rho_{*}^{k} \cdot I_{p}^{\text {raw }} \text { for all } p \in \mathcal{A}^{k},
$$

where $*$ refers to a normalisation approach (see Sec. IV-B). Demosaicing is then performed on the scale-adjusted raw image $I^{\prime r a w}$ to provide the estimated image $\hat{\mathbf{I}}^{\prime}$. After demosaicing, the post-normalisation step restores the original level scale of all pixels of each estimated channel $\hat{I}^{\prime k}$ as:

$$
\hat{I}_{p}^{k}=\frac{1}{\rho_{*}^{k}} \cdot \hat{I}_{p}^{\prime k} \text { for all } p \in \mathcal{A} .
$$

In the following we propose three ways to compute the normalisation factor $\rho_{*}^{k}$ of each channel. 


\section{B. Normalisation factors}

Equation (1) shows that image formation results from the product between the reflectance $R_{p}(\lambda)$, illumination $E(\lambda)$, and SRCs $S^{k}(\lambda)$ associated to the spectral bands. A first approach to limit the influence of illumination and camera sensitivity on the level ranges is to use the SRCs provided by the camera manufacturer (see Sec. IVB.1) and illumination properties (see Sec. IV-B.2) [22]. When there is no prior knowledge about the camera and illumination conditions, we propose to use the available levels in $I^{\text {raw }}$ in order to balance the level ranges (see Sec. IV-B.3).

1) Camera-based normalisation: When prior knowledge about the camera sensitivity is available, Lapray et al. [22] balance all SRCs $\left\{S^{k}(\lambda)\right\}_{k \in \mathcal{B}}$ so that the area defined by each of them over $\Omega$ is equal to 1 . We then propose the following normalisation factor $\rho_{c a m}^{k}$ based on camera properties:

$$
\rho_{c a m}^{k}=\frac{\max _{l \in \mathcal{B}} \sum_{\lambda=400}^{700} S^{l}(\lambda)}{\sum_{\lambda=400}^{700} S^{k}(\lambda)} .
$$

2) Camera- and illumination-based normalisation: When both the SRCs $\left\{S^{k}(\lambda)\right\}_{k \in \mathcal{B}}$ of the camera and the illumination $E(\lambda)$ of the scene are known, Lapray et al. [22] apply a scheme similar to a white balance on each channel. For this purpose the maximal energy that would be obtained from a perfect diffuser $(R(\lambda)=1$ for all $\lambda \in \Omega$ ) is divided by the energy obtained on each channel. We then propose the following normalisation factor $\rho_{c i}^{k}$ based on camera and illumination properties:

$$
\rho_{c i}^{k}=\frac{\max _{l \in \mathcal{B}} \sum_{\lambda=400}^{700} S^{l}(\lambda) E(\lambda)}{\sum_{\lambda=400}^{700} S^{k}(\lambda) E(\lambda)} .
$$

3) Raw image-based normalisation: In contrast with the two previous approaches, raw image-based normalisation does not use any prior knowledge about camera or illumination. We instead propose to balance the level ranges of all channels by only using the raw image levels [10]. For this purpose, we consider the ratio between the maximum level over all channels and the maximum level that is available for each channel in the raw image $I^{\text {raw }}$. The normalisation factor $\rho_{\text {raw }}^{k}$ is then given by:

$$
\rho_{\text {raw }}^{k}=\frac{\max _{s \in \mathcal{A}} I_{s}^{\text {raw }}}{\max _{s \in \mathcal{A}^{k}} I_{s}^{r a w}} .
$$

Note that this is similar to the max-spectral approach proposed by Khan et al. [23] for illumination estimation.

In the next section we assess the demosaicing performances reached when each of these three normalisation approaches is used.

\section{EXPERIMENTS}

To study the influence of illumination changes on the demosaicing performances, we compare the reference image $\mathbf{I}$ and its estimation $\hat{\mathbf{I}}$ for each demosaicing method described in Sec. III-A and each illumination of Fig. 1(a). For this purpose, we compute the peak signal-to-noise ratio (PSNR) on average over all channels as:

$$
\operatorname{PSNR}(\mathbf{I}, \hat{\mathbf{I}})=\frac{1}{K} \sum_{k \in \mathcal{B}} 10 \log _{10}\left(\frac{\left(\max _{p \in \mathcal{A}} I_{p}^{k}\right)^{2}}{M S E\left(I^{k}, \hat{I}^{k}\right)}\right),
$$


where $\operatorname{MSE}(\cdot, \cdot)$ denotes the mean squared error between two channels. Because $\max _{p \in \mathcal{A}} I_{p}^{k}$ can be low in case of low energy in the spectral band associated to channel $I^{k}$, Eq. (8) takes into account this actual maximal level rather than the theoretical one (255) to avoid misleading PSNR values.

Each method is assessed without and with the normalisation approaches proposed in Sec. IV-B. Tables 2(a) and 2(b) show the average PSNRs over CAVE and FOSTER databases, the best result for each illumination being highlighted as bold. They show that without normalisation the performances of all demosaicing methods are affected by illumination changes. Normalisation has no effect on images estimated by WB since this method only uses spatial correlation. Using camera-based normalisation $\left(\rho_{c a m}^{k}\right)$, the performances are fairly improved on illuminants E and D65 whose spectral power distribution is uniform. However, performances can be reduced in the case of illumination LD whose power distribution is located on three dominant narrow bands. Using camera- and illumination-based normalisation $\left(\rho_{c i}^{k}\right)$ provides the best performances for most of illuminations and methods. However, the illumination is unknown and has to be estimated when the camera is used in uncontrolled conditions. The same performances are practically reached by raw image-based normalisation $\left(\rho_{\text {raw }}^{k}\right)$ that does not require any prior knowledge about the camera or illumination. This simple approach which estimates these informations by using statistics of the raw image therefore gives satisfactory results whatever the demosaicing method and scene illumination conditions.

The best improvement provided by normalisation is reached using the PPID demosaicing method under HA illumination. For illustration purposes, we select an extract of size $125 \times 125$ pixels from the "Chart and stuffed toy" CAVE image simulated under the HA illumination. Reference and estimated images (using PPID) are converted to the sRGB colour space after integration by the rectangle method of the product between pixel levels and the CIE XYZ $2^{\circ}$ standard observer functions over the 16 band centres $\lambda^{k} \in \Lambda$. The results displayed in Fig. 4 show that the estimated image without normalisation presents severe zipper artefacts and false colours. Applying camera-based normalisation reduces those artefacts and the other two normalisation approaches slightly further improve the visual results.

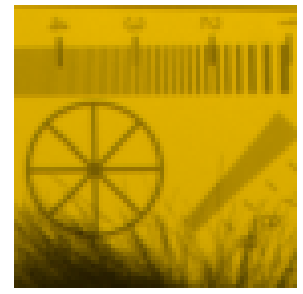

(a) Reference

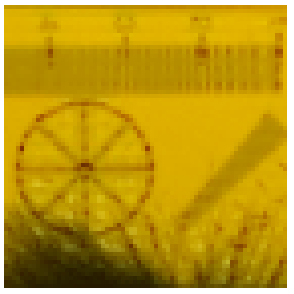

(b) None

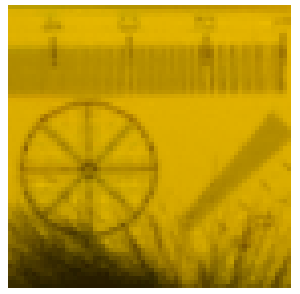

(c) $\rho_{c a m}^{k}$

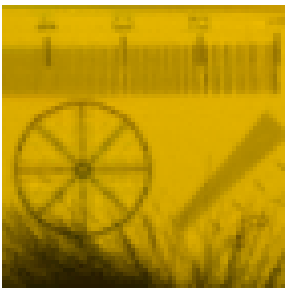

(d) $\rho_{c i}^{k}$

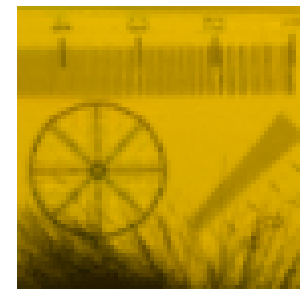

(e) $\rho_{\text {raw }}^{k}$

Fig. 4. sRGB renderings of a central extract of size $125 \times 125$ pixels from "Chart and stuffed toy" CAVE image simulated under HA illumination. (a) reference image, (b)-(e) images estimated by PPID demosaicing method with different normalisation approaches.

\section{Conclusion}

In this paper, we show that spectral correlation is fairly reduced in images acquired under illuminations that are non-homogeneous over the spectrum. This severely affects the performance of demosaicing schemes 
Table 2. PSNR (dB) of estimated images according to illumination (see Fig. 1(a)) (averages over all 32 CAVE (a) and 8 FOSTER (b) simulated images). Demosaicing methods: see Sec. III-A. Normalisations: camera-based $\rho_{c a m}^{k}$, camera- and illumination-based $\rho_{c i}^{k}$, raw image-based $\rho_{\text {raw }}^{k}$ (see Sec. IV-B).

(a) CAVE

\begin{tabular}{|c|c|c|c|c|c|c|c|}
\hline Method & Norm. & $\mathrm{E}$ & D65 & F12 & A & HA & LD \\
\hline \multirow{4}{*}{ WB } & \multirow{4}{*}{$\begin{array}{c}\text { None } \\
\rho_{c a m}^{k} \\
\rho_{c i}^{k} \\
\rho_{r a w}^{k} \\
\end{array}$} & 31.91 & 31.88 & 30.28 & 31.69 & 31.75 & 31.48 \\
\hline & & 31.91 & 31.88 & 30.28 & 31.69 & 31.76 & 31.48 \\
\hline & & 31.91 & 31.88 & 30.28 & 31.69 & 31.75 & 31.48 \\
\hline & & 31.91 & 31.88 & 30.28 & 31.69 & 31.76 & 31.48 \\
\hline \multirow{4}{*}{ PPIDWT } & \multirow{4}{*}{$\begin{array}{c}\text { None } \\
\rho_{c a m}^{k} \\
\rho_{c i}^{k} \\
\rho_{\text {raw }}^{k}\end{array}$} & 33.47 & 33.74 & 28.51 & 31.72 & 31.25 & 32.47 \\
\hline & & 35.48 & 35.42 & 30.69 & 34.50 & 34.15 & 32.18 \\
\hline & & 35.48 & 35.42 & 32.31 & 34.95 & 35.06 & 34.39 \\
\hline & & 35.42 & 35.36 & 32.25 & 34.89 & 35.01 & 34.34 \\
\hline \multirow{4}{*}{ PPIBTES } & \multirow{4}{*}{$\begin{array}{c}\text { None } \\
\rho_{c a m}^{k} \\
\rho_{c i}^{k} \\
\rho_{\text {raw }}^{k}\end{array}$} & 34.13 & 34.10 & 31.84 & 33.72 & 33.82 & 33.42 \\
\hline & & 34.29 & 34.23 & 31.99 & 33.94 & 34.04 & 33.43 \\
\hline & & 34.29 & 34.23 & 32.12 & 33.99 & 34.12 & 33.58 \\
\hline & & 34.29 & 34.23 & 32.12 & 33.99 & 34.12 & 33.58 \\
\hline \multirow{4}{*}{ SD } & \multirow{4}{*}{$\begin{array}{c}\text { None } \\
\rho_{c a m}^{k} \\
\rho_{c i}^{k} \\
\rho_{\text {raw }}^{k}\end{array}$} & 33.80 & 34.02 & 29.23 & 32.26 & 32.02 & 32.68 \\
\hline & & 35.30 & 35.23 & 31.19 & 34.51 & 34.36 & 32.70 \\
\hline & & 35.30 & 35.24 & 32.29 & 34.80 & 34.94 & 34.30 \\
\hline & & 35.25 & 35.19 & 32.20 & 34.75 & 34.89 & 34.26 \\
\hline \multirow{4}{*}{ MLDI } & \multirow{4}{*}{$\begin{array}{c}\text { None } \\
\rho_{c a m}^{k} \\
\rho_{c i}^{k} \\
\rho_{r a w}^{k} \\
\end{array}$} & 36.95 & 37.17 & 31.37 & 34.99 & 34.70 & 35.50 \\
\hline & & 38.71 & 38.60 & 33.31 & 37.54 & 37.50 & 35.24 \\
\hline & & 38.71 & 38.59 & 34.32 & 37.86 & 38.14 & 37.02 \\
\hline & & 38.68 & 38.56 & 34.28 & 37.84 & 38.12 & 36.99 \\
\hline \multirow{4}{*}{ PPID } & \multirow{4}{*}{$\begin{array}{c}\text { None } \\
\rho_{c a m}^{k} \\
\rho_{c i}^{k} \\
\rho_{r a w}^{k} \\
\end{array}$} & 36.71 & 37.06 & 30.32 & 34.36 & 34.08 & 34.71 \\
\hline & & 39.84 & 39.65 & 32.50 & 37.89 & 37.60 & 34.73 \\
\hline & & 39.84 & 39.69 & 34.18 & 38.49 & 38.81 & $\mathbf{3 7 . 4 6}$ \\
\hline & & 39.74 & 39.59 & 34.10 & 38.40 & 38.73 & 37.40 \\
\hline
\end{tabular}

(b) FOSTER

\begin{tabular}{|c|c|c|c|c|c|c|c|}
\hline Method & Norm. & E & D65 & F12 & A & HA & LD \\
\hline \multirow{4}{*}{ WB } & \multirow{4}{*}{$\begin{array}{c}\text { None } \\
\rho_{c a m}^{k} \\
\rho_{c i}^{k} \\
\rho_{r a w}^{k} \\
\end{array}$} & 33.09 & 33.13 & 29.83 & 32.27 & 32.20 & 32.50 \\
\hline & & 33.10 & 33.13 & 29.84 & 32.27 & 32.20 & 32.51 \\
\hline & & 33.10 & 33.13 & 29.83 & 32.27 & 32.20 & 32.50 \\
\hline & & 33.09 & 33.13 & 29.83 & 32.27 & 32.20 & 32.51 \\
\hline \multirow{4}{*}{ PPIDWT } & \multirow{4}{*}{$\begin{array}{c}\text { None } \\
\rho_{c a m}^{k} \\
\rho_{c i}^{k} \\
\rho_{\text {raw }}^{k} \\
\end{array}$} & 33.09 & 33.46 & 27.02 & 30.88 & 30.28 & 31.99 \\
\hline & & 36.30 & 36.27 & 29.05 & 34.44 & 33.78 & 32.19 \\
\hline & & 36.30 & 36.22 & 31.12 & 35.29 & 35.41 & 34.64 \\
\hline & & 36.39 & 36.31 & 31.09 & 35.28 & 35.42 & 34.71 \\
\hline \multirow{4}{*}{ PPIBTES } & \multirow{4}{*}{$\begin{array}{c}\text { None } \\
\rho_{c a m}^{k} \\
\rho_{c i}^{k} \\
\rho_{r a w}^{k} \\
\end{array}$} & 34.49 & 34.53 & 30.59 & 33.45 & 33.39 & 33.63 \\
\hline & & 34.80 & 34.80 & 30.77 & 33.82 & 33.77 & 33.65 \\
\hline & & 34.80 & 34.79 & 30.96 & 33.91 & 33.90 & 33.91 \\
\hline & & 34.81 & 34.80 & 30.96 & 33.91 & 33.91 & 33.91 \\
\hline \multirow{4}{*}{ SD } & \multirow{4}{*}{$\begin{array}{c}\text { None } \\
\rho_{c a m}^{k} \\
\rho_{c i}^{k} \\
\rho_{\text {raw }}^{k}\end{array}$} & 33.93 & 34.26 & 28.09 & 31.81 & 31.44 & 32.62 \\
\hline & & 36.31 & 36.27 & 29.89 & 34.71 & 34.29 & 33.04 \\
\hline & & 36.31 & 36.25 & 31.17 & 35.25 & 35.32 & 34.83 \\
\hline & & 36.38 & 36.32 & 31.10 & 35.24 & 35.33 & 34.88 \\
\hline \multirow{4}{*}{ MLDI } & \multirow{4}{*}{$\begin{array}{c}\text { None } \\
\rho_{c a m}^{k} \\
\rho_{c i}^{k} \\
\rho_{r a w}^{k} \\
\end{array}$} & 36.44 & 36.79 & 29.84 & 33.96 & 33.54 & 35.01 \\
\hline & & 39.28 & 39.20 & 31.61 & 37.16 & 36.90 & 35.06 \\
\hline & & 39.28 & 39.16 & 32.66 & 37.74 & 37.96 & 37.06 \\
\hline & & 39.31 & 39.19 & 32.60 & 37.73 & 37.96 & 37.07 \\
\hline \multirow{4}{*}{ PPID } & \multirow{4}{*}{$\begin{array}{c}\text { None } \\
\rho_{c a m}^{k} \\
\rho_{c i}^{k} \\
\rho_{\text {raw }}^{k}\end{array}$} & 35.31 & 35.70 & 28.18 & 32.70 & 32.26 & 33.36 \\
\hline & & 38.95 & 38.80 & 30.17 & 36.57 & 36.03 & 33.80 \\
\hline & & 38.95 & 38.77 & 31.96 & 37.44 & 37.71 & 36.29 \\
\hline & & 39.04 & 38.86 & 31.92 & 37.42 & 37.71 & 36.35 \\
\hline
\end{tabular}


that mainly rely on assumptions about spectral correlation. To overcome this limitation, we propose to insert normalisation steps in the imaging pipeline to adjust channel levels before demosaicing and restore them afterwards. The channel-specific normalisation factor can be deduced either from the SRCs of the camera, from the SRCs and the illumination, or directly estimated from the raw image levels. Experimental results show that normalisation based on the sole SRCs provides good but illumination-sensitive results. Normalisation based on SRCs and illumination informations provides the best results despite illumination is not always available in practice. At last raw image-based normalisation provides promising results without any a priori knowledge about the camera or illumination, and thus constitutes a good compromise for demosaicing. Future work will focus on the assessment of these approaches with other cameras and on experiments with sensor noise consideration.

\section{ACKNOWLEDGMENT}

This work was supported (in part) by the National French project CNRS-INS2I-JCJC-2017 MOSAIC and by the ANR-11-EQPX-23 IrDIVE platform.

\section{REFERENCES}

[1] J. Pichette, A. Laurence, L. Angulo, F. Lesage, A. Bouthillier, D. Nguyen, and F. Leblond, "Intraoperative video-rate hemodynamic response assessment in human cortex using snapshot hyperspectral optical imaging," Neurophotonics, vol. 3, no. 4, p. 045003, Oct. 2016.

[2] X. Hadoux, N. Gorretta, J.-M. Roger, R. Bendoula, and G. Rabatel, "Comparison of the efficacy of spectral pre-treatments for wheat and weed discrimination in outdoor conditions," Computers and Electronics in Agriculture, vol. 108, pp. 242-249, 2014.

[3] M. Elias and P. Cotte, "Multispectral camera and radiative transfer equation used to depict Leonardo's sfumato in Mona Lisa," Applied Optics, vol. 47, no. 12, pp. 2146-2154, Apr. 2008.

[4] C. Liu, W. Liu, X. Lu, F. Ma, W. Chen, J. Yang, and L. Zheng, "Application of multispectral imaging to determine quality attributes and ripeness stage in strawberry fruit," PLOS ONE, vol. 9, no. 2, pp. 1-8, Feb. 2014.

[5] S. Serranti, A. Gargiulo, and G. Bonifazi, "Classification of polyolefins from building and construction waste using NIR hyperspectral imaging system," Resources, Conservation and Recycling, vol. 61, pp. 52-58, Apr. 2012.

[6] R. Arablouei, E. Goan, S. Gensemer, and B. Kusy, "Fast and robust pushbroom hyperspectral imaging via DMD-based scanning," in Procs. SPIE: Novel Optical Systems Design and Optimization XIX, vol. 9948, San Diego, CA, USA, Aug. 2016, pp. 99 480A-99480A-11.

[7] L. Gao and L. V. Wang, "A review of snapshot multidimensional optical imaging: Measuring photon tags in parallel," Physics Reports, vol. 616, pp. 1-37, Feb. 2016.

[8] X. Cao, T. Yue, X. Lin, S. Lin, X. Yuan, Q. Dai, L. Carin, and D. J. Brady, "Computational snapshot multispectral cameras: Toward dynamic capture of the spectral world," IEEE Signal Process. Mag., vol. 33, no. 5, pp. 95-108, Sep. 2016. 
[9] J.-B. Thomas, P.-J. Lapray, P. Gouton, and C. Clerc, "Spectral characterization of a prototype SFA camera for joint visible and NIR acquisition," Sensors, vol. 16, no. 7, p. 993, Jun. 2016.

[10] S. Mihoubi, O. Losson, B. Mathon, and L. Macaire, "Multispectral demosaicing using pseudo-panchromatic image," IEEE Trans. Comput. Imaging, vol. PP, no. 99, pp. 1-1, 2017.

[11] F. Yasuma, T. Mitsunaga, D. Iso, and S. K. Nayar, "Generalized assorted pixel camera: Postcapture control of resolution, dynamic range, and spectrum,” IEEE Trans. Image Process., vol. 19, no. 9, pp. 2241-2253, Sep. 2010.

[12] D. H. Foster, K. Amano, S. M. C. Nascimento, and M. J. Foster, "Frequency of metamerism in natural scenes," Journal of the Optical Society of America A, vol. 23, no. 10, pp. 2359-2372, Oct 2006.

[13] B. Geelen, N. Tack, and A. Lambrechts, "A compact snapshot multispectral imager with a monolithically integrated per-pixel filter mosaic," in Procs. SPIE: Advanced Fabrication Technologies for Micro/Nano Optics and Photonics VII, vol. 8974, San Francisco, CA, USA, Feb. 2014, pp. 89740L-89740L-8.

[14] B. K. Gunturk, Y. Altunbasak, and R. M. Mersereau, "Color plane interpolation using alternating projections," IEEE Trans. Image Process., vol. 11, no. 9, pp. 997-1013, Sep. 2002.

[15] N.-X. Lian, L. Chang, V. Zagorodnov, and Y.-P. Tan, "Reversing demosaicking and compression in color filter array image processing: Performance analysis and modeling," IEEE Trans. Image Process., vol. 15, no. 11, pp. 3261-3278, Nov. 2006.

[16] R. Zhen and R. L. Stevenson, Color Image and Video Enhancement. Springer, 2015, ch. Image Demosaicing, pp. 13-54.

[17] J. Brauers and T. Aach, "A color filter array based multispectral camera," in Institute of Imaging and Computer Vision, RWTH Aachen University, 52056 Aachen, Germany, Oct. 2006.

[18] L. Miao, H. Qi, R. Ramanath, and W. Snyder, "Binary tree-based generic demosaicking algorithm for multispectral filter arrays," IEEE Trans. Image Process., vol. 15, no. 11, pp. 3550-3558, Nov. 2006.

[19] X. Wang, J.-B. Thomas, J. Y. Hardeberg, and P. Gouton, "Discrete wavelet transform based multispectral filter array demosaicking," in Procs. 2013 Colour and Visual Computing Symposium (CVCS 2013), Gjøvik, Norway, Sep. 2013, pp. 1-6.

[20] S. Mihoubi, O. Losson, B. Mathon, and L. Macaire, "Multispectral demosaicing using intensity in edgesensing and iterative difference-based methods," in Procs. 12th International Conference on Signal-Image Technology \& Internet-based Systems (SITIS'16), Naples, Italy, Nov. 2016, pp. 805-810.

[21] K. Shinoda, S. Ogawa, Y. Yanagi, M. Hasegawa, S. Kato, M. Ishikaway, H. Komagatay, and N. Kobayashi, "Multispectral filter array and demosaicking for pathological images," in Procs. APSIPA Annual Summit and Conference 2015, Hong Kong, China, Dec. 2015, pp. 697-703.

[22] P. Lapray, J.-B. Thomas, P. Gouton, and Y. Ruichek, "Energy balance in Spectral Filter Array camera design," Journal of the European Optical Society-Rapid Publications, vol. 13, no. 1, Jan. 2017.

[23] H. A. Khan, J.-B. Thomas, J. Y. Hardeberg, and O. Laligant, "Illuminant estimation in multispectral imaging," Journal of the Optical Society of America A, vol. 34, no. 7, pp. 1085-1098, Jul. 2017. 